\title{
Highly Sensitive Refractive Index Sensor Based on Plasmonic Bow Tie Configuration
}

\author{
Muhammad Ali BUTT ${ }^{1 *}$, Nikolay Lvovich KAZANSKIY ${ }^{1,2}$, \\ and Svetlana Nikolaevna KHONINA ${ }^{1,2}$ \\ ${ }^{1}$ Department of Technical Cybernetic, Samara National Research University, Samara 443086, Russia \\ ${ }^{2}$ Institute of RAS-Branch of the FSRC "Crystallography and Photonics", RAS, 151 Molodogvardeiskaya, Samara \\ 443001, Russia \\ *Corresponding author: Muhammad Ali BUTT \\ E-mail: ali_ciit_engineer@yahoo.com
}

\begin{abstract}
We propose a highly refractive index sensor based on plasmonic Bow Tie configuration. The sensitivity of the resonator design is enhanced by incorporating a nanowall (NW) in a modified Bow Tie design where sharp tips of $\mathrm{V}$-junction are flattened. This approach provides high confinement of electric field distribution of surface plasmon polariton (SPP) mode in the narrow region of the cavity. Consequently, the effective refractive index ( $\left.n_{\text {eff }}\right)$ of the mode increases and is highly responsive to the ambient medium. The sensitivity analysis of the SPP mode is calculated for six resonator schemes. The results suggest that the NW embedded cavity offers the highest mode sensitivity due to the large shift of effective index when exposed to a slight change in the medium refractive index. Moreover, the device sensitivity of the proposed design is approximated at $2300 \mathrm{~nm} / \mathrm{RIU}$ which is much higher than the sensitivity of the standard Bow Tie configuration.
\end{abstract}

Keywords: Bow Tie configuration; plasmonic sensor; metal-insulator-metal waveguide; refractive index sensor

Citation: Muhammad Ali BUTT, Nikolay Lvovich KAZANSKIY, and Svetlana Nikolaevna KHONINA, "Highly Sensitive Refractive Index Sensor Based on Plasmonic Bow Tie Configuration," Photonic Sensors, 2020, 10(3): 223-232.

\section{Introduction}

Surface plasmon polaritons (SPPs) are electromagnetic waves propagating along with the metal-dielectric interface with an exponentially decaying field and have an evolving apprehension of highly integrated optical circuits due to their capability to overcome the diffraction limit of light $[1,2]$. For SPPs-based photonic devices, the metal-insulator-metal (MIM) waveguide scheme is one of the fundamental structures. MIM waveguides are plasmonic structures in which an insulator is enclosed by two metal claddings [3, 4]. Recent developments of plasmonic devices based on the
MIM waveguide have proven their effectiveness for subwavelength manipulation of light with an acceptable propagation length due to the guided wave surface plasmon resonance (SPR) modes $[5,6]$. To realize highly integrated optical circuits, intensive research has been conducted on the design of a diverse range of devices using MIM waveguides. These devices incorporate couplers [7], filters [8, 9], sensors [10-12], demultiplexers [13], modulators [14], switches [15], and splitters [16], among others.

Refractive index sensors have several applications in the biological and chemical fields and have been intensively studied in recent years, 
for example, the solution concentration and $\mathrm{pH}$ value can be measured via refractive index changes [17-20]. When a substance under investigation is brought into contact with the sensor, $n_{\text {eff }}$ of the MIM waveguide changes, and as a result, $\lambda_{\text {res }}$ undergoes a redshift. Surface plasmons (SPs) are highly sensitive to changes in the ambient refractive index which is determined by measuring the changes in one of the characteristics of the light coupled to the SP, for instance, changes in $\lambda_{\text {res}}$, intensity, or phase. Recently, several plasmonic sensor configurations are proposed for refractive index sensing applications. The main intention behind such diversity in designs is to enhance the device sensitivity to measure a slight change in the ambient refractive index. There are several examples of recently proposed MIM waveguide plasmonic sensors presented in Table 1.

In this work, we propose Bow Tie cavity configurations which provide a strong SPP mode confinement in the narrow region. As a result, there is a high light-mater interaction which results in the enhanced sensitivity of the sensor. The transmission spectrum and E-field distributions are simulated using COMSOL 5.1 software by utilizing a 2D (two-dimension)-finite element method (2D-FEM). The EM-wave frequency domain (emw) is chosen as the physics interface and the modal analysis is added to the study. In COMSOL simulations, the subdomains of the sensor design are divided into triangular mesh element with a fine mesh grid size for the air medium and waveguide geometries. This allows us to obtain precise simulation results within the available computational resources. When solving EM wave problems, it is advantageous to model a domain with open boundaries that is a boundary of the computational domain through which an EM wave can pass without reflection. To approximate an open geometry, scattering boundary conditions (SBC) are used at the outer edges of the FEM simulation window.
Table 1 Recently proposed MIM WG based resonators.

\begin{tabular}{|c|c|c|c|}
\hline & Sensor design & $\begin{array}{l}\text { Sensitivity } \\
(\mathrm{nm} / \mathrm{RIU})\end{array}$ & $\begin{array}{l}\text { Figure of merit } \\
\qquad(F O M)\end{array}$ \\
\hline$[21]$ & Tangent ring & 880 & 964 \\
\hline$[22]$ & Concentric rings & 1060 & 203.8 \\
\hline$[23]$ & Nano-dots embedded ring & 907 & 40.8 \\
\hline$[24]$ & Hexagon cavity & 1562.5 & 38.6 \\
\hline$[25]$ & Isosceles triangular cavity & 1200 & $3 \times 10^{6}\left(F O M^{*}\right)$ \\
\hline$[26]$ & $\begin{array}{l}\text { Notched ring cavity and } \\
\text { stub }\end{array}$ & 1071.4 & 14.29 \\
\hline$[27]$ & Asymmetric MIM WG & 718 & $\begin{array}{c}\text { Not mentioned in } \\
\text { the paper }\end{array}$ \\
\hline$[28]$ & $\begin{array}{l}\text { Double rectangular } \\
\text { cavities }\end{array}$ & 596 & $\begin{array}{c}\text { Not mentioned in } \\
\text { the paper }\end{array}$ \\
\hline [29] & Symmetric MIM WG & 1268 & 280 \\
\hline$[30]$ & Circular ring resonator & $300.0-868.4$ & $\begin{array}{c}\text { Not mentioned in } \\
\text { the paper }\end{array}$ \\
\hline [31] & $\begin{array}{l}\text { Vertical split-ring } \\
\text { resonator }\end{array}$ & 1400 & $\begin{array}{c}\text { Not mentioned in } \\
\text { the paper }\end{array}$ \\
\hline$[32]$ & Multi split-ring resonator & 793.3 & 52.9 \\
\hline$[33]$ & Two stubs & 2000 & $\begin{array}{c}4.05 \times 10^{4} \\
\left(F O M^{*}\right)\end{array}$ \\
\hline
\end{tabular}

Note: In some works such as [25, 33], FOM is exceedingly high and is represented as $F O M^{*}$ which is calculated differently using the expression $\Delta R /(R \Delta n)$ at a fixed wavelength, where $\Delta R$ denotes the reflection intensity variation due to the change in the refractive index $(\Delta n)$ of the surrounding medium and $R$ is the reflection rate in the sensor structure. It can also be calculated by using the expression $\Delta T /(T \Delta n)$, where $T$ denotes the transmittance in the proposed structures and $\Delta T / \Delta n$ is the transmission change at a fixed wavelength induced by a refractive index change.

\section{Sensor design}

At first, a Bow Tie (BT) resonator configuration is studied which is formed by compressing a square air cavity from the middle. This approach provides an E-field enhancement of the SPP mode in a narrow region which results in a better light-matter interaction. This effect is mainly observed if the nano metallic dot/wall is introduced inside this narrow region. Therefore, several BT design configurations are investigated to further enhance SPPs confinement. This includes nanodot (ND) and nanowall (NW) embedded BT configuration. The width of the bus waveguide, the side length of the cavity, the gap in the cavity, and separation between bus waveguide and the cavity are denoted by $w, L, g$, and $d$, respectively. The schematic of a standard BT scheme is shown in Fig. 1(a).

The metal and insulator materials are defined as silver and air, respectively. Silver is preferred due to 
its EM response within near-infrared (NIR) which has the negligible imaginary part of relative permittivity. As a result, its power consumption is low as compared with gold and copper. The frequencydependent complex relative permittivity of silver, $\varepsilon_{m}$, is characterized by the Lorentz-Drude model [34].

$$
\varepsilon_{m}(\omega)=\varepsilon_{\infty}-\frac{\omega_{p}^{2}}{\omega(\omega+\mathrm{i} \gamma)}
$$

where $\varepsilon_{\infty}=3.7$ is the dielectric constant at an infinite frequency, $\omega_{p}=9.1 \mathrm{eV}$ is the bulk plasma frequency of free conduction electrons, $\gamma=0.018 \mathrm{eV}$ is the electron collision frequency, and $\omega$ is the angular frequency of incident light in vacuum. The TM mode of the MIM bus waveguide is excited by a plane wave and the transmission spectrum is obtained by a line integration of the output port. As $w<<\lambda_{\text {incident }}$, therefore, only the fundamental TM mode can exist. The dispersion relation of this fundamental mode can be written as

$$
\begin{gathered}
\frac{\varepsilon_{d} p}{\varepsilon_{m} k}=\frac{1-\mathrm{e}^{k w}}{1+\mathrm{e}^{k w}} \\
k=k_{o} \sqrt{\left(\frac{\beta_{\mathrm{SPP}}}{k_{o}}\right)^{2}-\varepsilon_{d}}, p=k_{o} \sqrt{\left(\frac{\beta_{\mathrm{SPP}}}{k_{o}}\right)^{2}-\varepsilon_{m}} \\
\beta_{\mathrm{SPP}}=n_{\mathrm{eff}} k_{o}=n_{\mathrm{eff}} \frac{2 \pi}{\lambda}
\end{gathered}
$$

where $w$ is the width of the bus WG, $\lambda$ is the incident light wavelength in vacuum, $\varepsilon_{d}$ and $\varepsilon_{m}$ give the relative dielectric and metal permittivity, $\beta_{\mathrm{SPP}}$ and $n_{\text {eff }}$ are the propagation constant and effective refractive index of SPPs, respectively, and $k_{o}=2 \pi / \lambda$ is the wavenumber.

A typical transmission spectrum of the sensor is displayed in Fig. 1(b), exhibiting two sharp resonance dips at $852 \mathrm{~nm}$ and $1708 \mathrm{~nm}$. The geometric parameters of the design are $w=50 \mathrm{~nm}, L=350 \mathrm{~nm}$, $d=30 \mathrm{~nm}$, and $g=30 \mathrm{~nm}$. The corresponding E-field distribution $|\mathbf{E}|$ for both modes, obtained from FEM is displayed in the inset of Fig. 1(b). The 3D electric field intensity profile is shown in Fig. 1(c) which shows the strong SPP mode confinement at two points in the middle of the cavity. We are concerned about the resonance mode which provides a strong E-field confinement in the region where the cavity is compressed. As a result, this SPP mode exhibits a high effective refractive index $\left(n_{\text {eff }}\right)$ which is highly sensitive to the ambient medium.

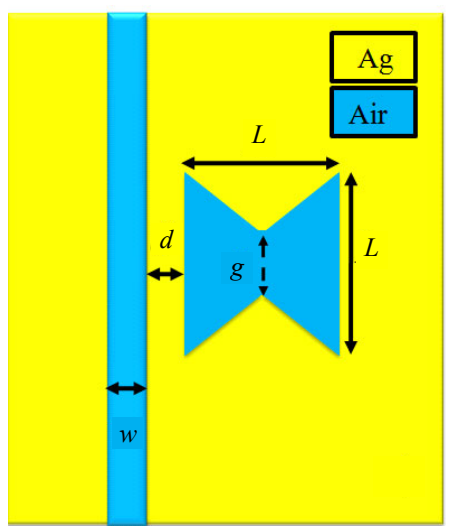

(a)

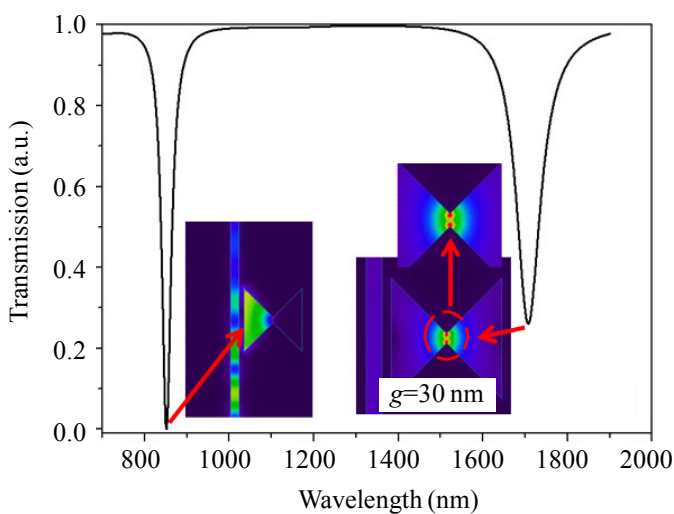

(b)

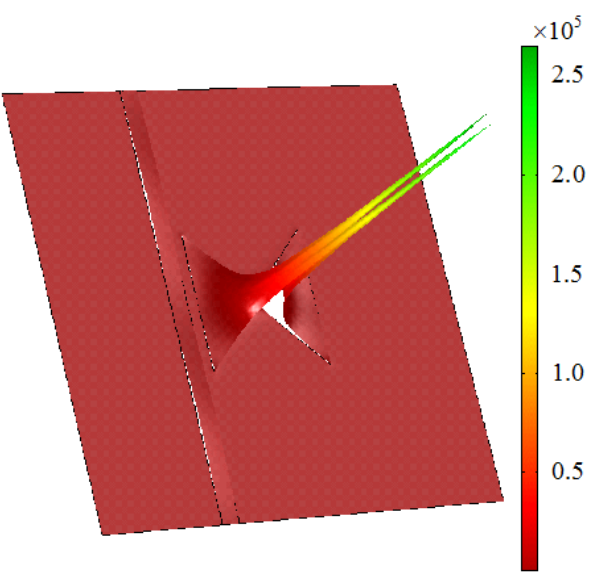

(c)

Fig. 1 Schematic and E-field distribution: (a) standard BT scheme, (b) transmission spectrum of a BT resonator at $n=1.0$ (inset shows the E-field distribution of two resonant dips), and (c) $3 \mathrm{D}$ electric field intensity distribution in the cavity at the resonance wavelength. 


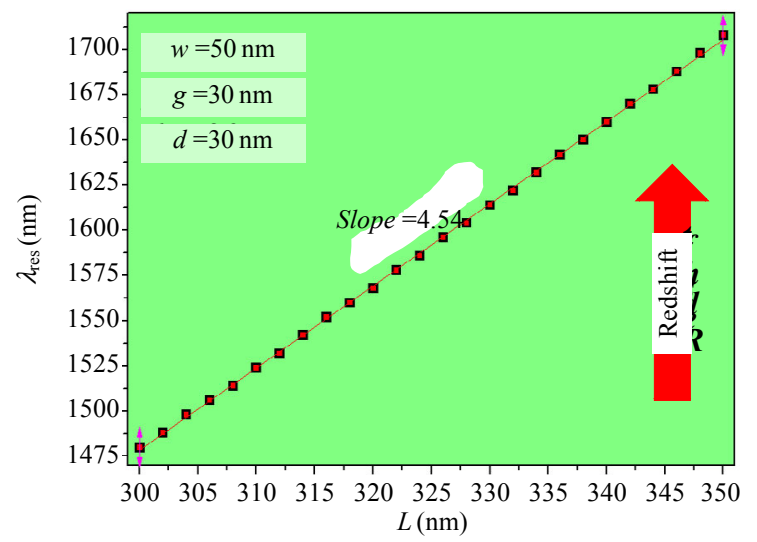

(a)

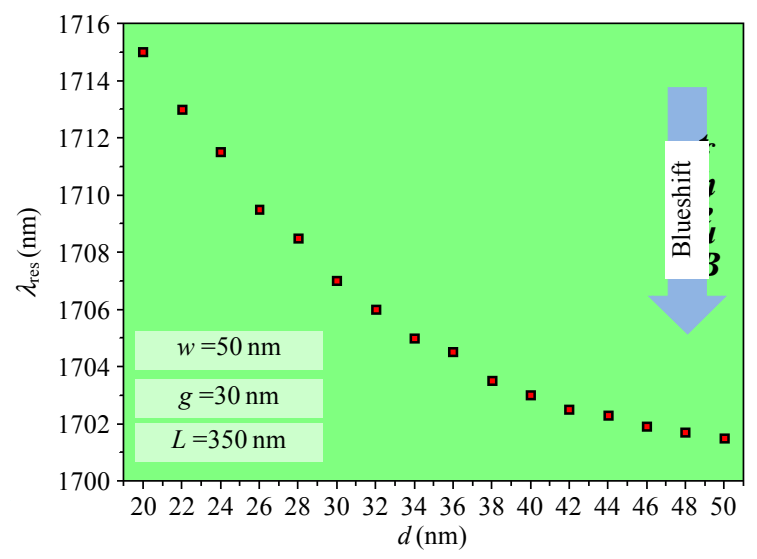

(b)

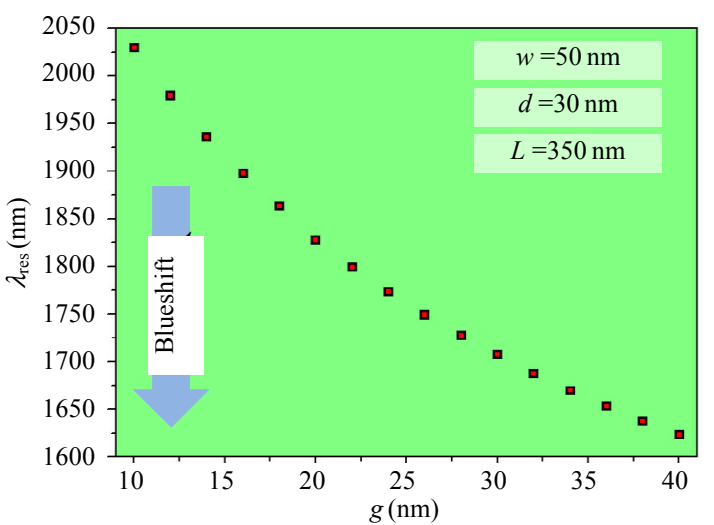

(c)

Fig. 2 Dependence of the resonance wavelength on (a) BT length, (b) separation between the bus WG and BT resonator, and (c) BT cavity gap.

The transmission characteristics of the plasmonic waveguide system can be affected by the structural parameters. That is why the spectral characteristics of the sensor design are investigated which is extremely tunable by manipulating the dimensions of the device. Therefore, the dependence of $\lambda_{\text {res }}$ on the geometric parameters of the sensor such as $L, g$, and $d$ is studied. Figure2(a) shows the dependence of $\lambda_{\text {res }}$ on $L$ which is varied from $300 \mathrm{~nm}$ to $350 \mathrm{~nm}$, whereas other parameters such as $g, d$, and $w$ are fixed at $30 \mathrm{~nm}, 30 \mathrm{~nm}$, and $50 \mathrm{~nm}$, respectively. It is worth noting that there is a direct relationship between $\lambda_{\text {res }}$ and $L$. However, $d$ and $g$ have an indirect relationship with $\lambda_{\text {res }}$ that is why a blueshift in $\lambda_{\text {res }}$ is observed with an increasing value of $g$ and $d$ as shown in Figs. 2(b) and 2(c). The parameters used in Fig. 2(b) are $w=50 \mathrm{~nm}, g=30 \mathrm{~nm}$, and $L=350 \mathrm{~nm}$, whereas in Fig. 2(c), the parameters are $w=50 \mathrm{~nm}$, $d=30 \mathrm{~nm}$, and $L=350 \mathrm{~nm}$.

\section{ND and NW embedded BT configuration}

In this section, we model four different BT configurations by incorporating ND and NW in the region where the cavity of $\mathrm{BT}$ is narrowed. This approach allows further enhancement of E-field of the SPP mode between the boundaries of BT and ND/NW. The spectral characteristics of all the BT design configurations are compared in the last section of the paper.

\subsection{ND embedded standard BT configuration}

In this configuration, a single silver ND is integrated into the center of the standard BT configuration as shown in Fig. 3(a). This arrangement allows the strong confinement of SPPs 
between the boundaries of ND and sharp tips of BT as shown in Fig. 3(e). The radius of ND is denoted by $R_{\mathrm{ND}}=15 \mathrm{~nm}$ while the gap between the upper and lower boundaries of the ND and a sharp tips of BT is denoted by $g 1$ and $g 2$, respectively, which is kept at $5 \mathrm{~nm}$. Whereas, all the other design parameters are

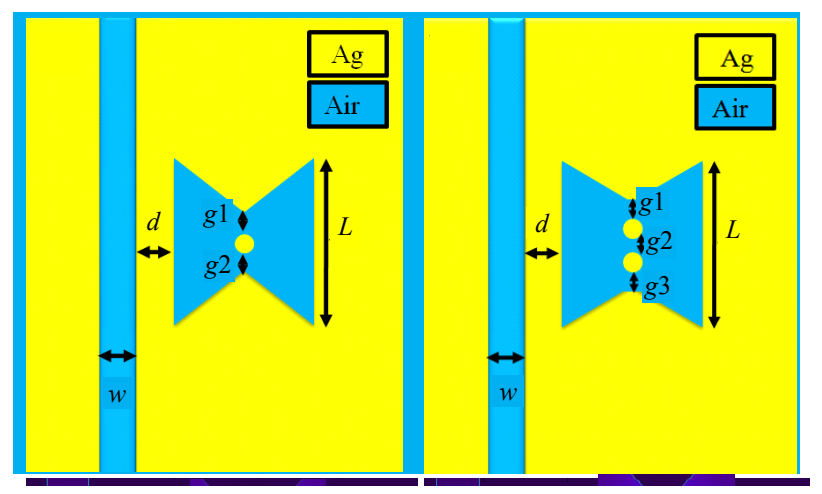

(a)

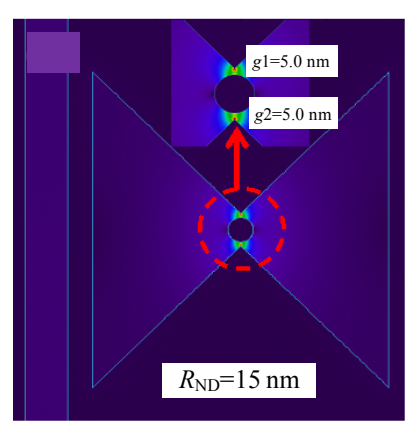

(e) (b)

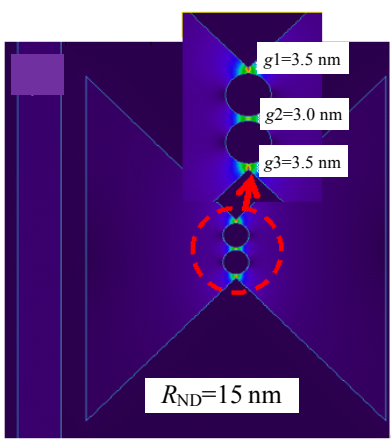

(f) the same as the standard BT design. The size of the ND plays an important role in the enhancement of the E-field in the narrow region of the cavity. The spectral characteristics of the ND embedded BT resonator are studied for $R_{\mathrm{ND}}=10 \mathrm{~nm}$ and $15 \mathrm{~nm}$, discussed in Section 4.

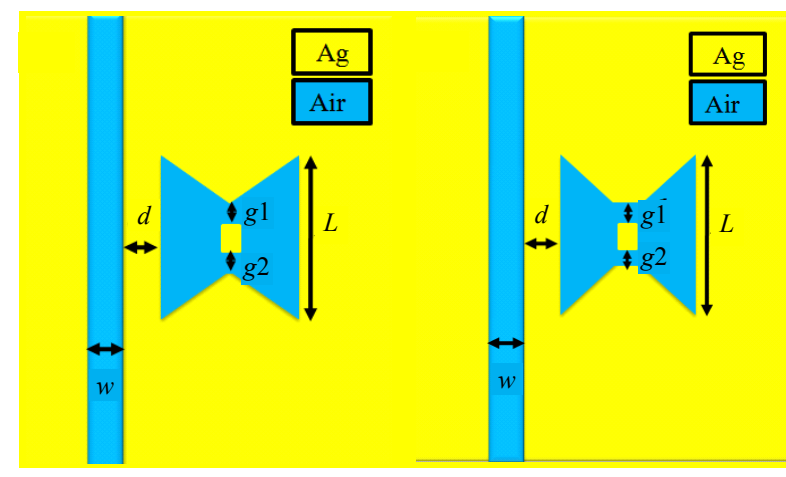

(c)

(d)

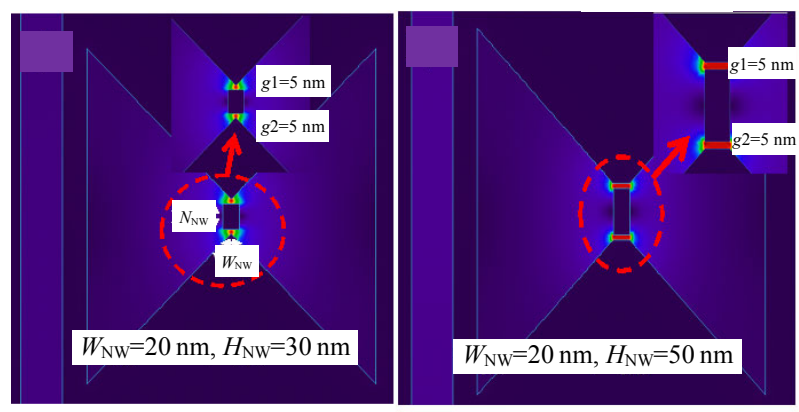

(g)

(h)

Fig. 3 Schematic of (a) ND incorporated in the standard BT scheme, (b) two NDs vertically embedded in the standard BT scheme, (c) NW incorporated in the standard BT scheme, (d) NW incorporated flat boundaries BT scheme, and (e)-(h) E-field distributions in design configurations as mentioned in (a), (b), (c) and (d), respectively.

\subsection{Two vertically arranged NDs embedded standard BT configuration}

Here, we consider two silver NDs vertically arranged in the center of the standard BT design as shown in Fig. 3(b). The gap between the upper and lower boundaries of the ND and sharp tips of BT is denoted by $g 1$ and $g 3$, respectively, whereas, $g 2$ is the gap between two NDs. The $R_{\mathrm{ND}}, g 1, g 2$, and $g 3$ are fixed at $15 \mathrm{~nm}, 3.5 \mathrm{~nm}, 3.0 \mathrm{~nm}$, and $3.5 \mathrm{~nm}$, respectively, where $2 \mathrm{NDs}+g 1+g 2+g 3=70 \mathrm{~nm}$. Figure3(f) shows the E-field distribution of the SPP mode in three different regions which can provide strong light-matter interaction.

\subsection{NW embedded standard BT configuration}

In this configuration, silver NW is placed in the center of the standard BT design as shown in Fig. $3(\mathrm{c})$. The height and width of the NW are denoted by $H_{\mathrm{NW}}$ and $W_{\mathrm{NW}}$ which are equal to $30 \mathrm{~nm}$ and $20 \mathrm{~nm}$, respectively, where $g 1$ and $g 2$ are the gaps between the upper and lower boundaries of the NW and sharp tips of the BT. The geometry of the NW facilitates the strong SPP modal confinement on the 
surface of the nano metallic wall. The E-field distribution of the SPP mode in the BT design at $\lambda_{\text {res }}$ is shown in Fig. $3(\mathrm{~g})$, where the total gap $\left(H_{\mathrm{NW}}+g 1+g 1=30 \mathrm{~nm}+5 \mathrm{~nm}+5 \mathrm{~nm}\right)$ between two sharp tips of the BT is $40 \mathrm{~nm}$.

\subsection{NW embedded modified BT configuration}

Last but not least, silver NW is embedded in the center of the modified BT design as shown in Fig. 3(d). The sharp tips of the BT V-junction are flattened (width $=20 \mathrm{~nm}$ ) to increase the surface area between the NW and boundaries of the BT. An NW of $H_{\mathrm{NW}}=50 \mathrm{~nm}$ and $W_{\mathrm{NW}}=20 \mathrm{~nm}$ is placed in the middle of the cavity of $g=60 \mathrm{~nm}$. As a result, $5 \mathrm{~nm}$ of the slot is created on the top and bottom of the NW which are represented as $g 1$ and $g 2$, respectively. When the resonance condition is fulfilled, highly sensitive SPP modes can be confined in these slots. The remaining geometric parameters of the resonator such as $L, d$, and $w$ are fixed at $350 \mathrm{~nm}, 30 \mathrm{~nm}$, and $50 \mathrm{~nm}$, respectively.

In this configuration, $n_{\text {eff }}$ of the SPP mode is quite high which is highly responsive to the ambient medium. A small change in the medium refractive index can bring an enormous shift in $\lambda_{\text {res. }}$. The spectral characteristics of the sensor design are discussed in the next section. The E-field distribution of the SPP mode around the NW is displayed in Fig. 3(h) where $W_{\mathrm{NW}}$ and $H_{\mathrm{NW}}$ are fixed at $20 \mathrm{~nm}$ and $50 \mathrm{~nm}$, respectively.

For sensing applications, SPP field sensitivity $\left(S_{\mathrm{SPP}}\right)$ is an essential parameter which should be taken into consideration while designing sensing devices. We evaluate the $S_{\mathrm{SPP}}$ of six different schemes of BT resonator $S_{\mathrm{SPP}}$ by using the following expression:

$$
S_{\mathrm{SPP}}=\frac{n_{\text {eff-sensing }}-n_{\text {eff-air }}}{n_{\text {sensing }}-n_{\text {air }}}
$$

where $n_{\text {eff-sensing }}$ is the effective refractive index at the refractive index of the analyte ( $\left.n_{\text {sensing }}\right)$ and $n_{\text {eff-air }}$ is the effective refractive index at the refractive index of air $\left(n_{\text {air }}=1.0\right)$. In this analysis, $n_{\text {sensing }}=1.01-1.1$ is used to calculate the sensitivity of SPP mode. The geometric parameters of the BT resonator are presented in Table2.

Table 2 Geometric parameters of the BT resonator.

\begin{tabular}{|c|c|c|c|c|c|}
\hline Scheme 1 & Scheme 2 & Scheme 3 & Scheme 4 & Scheme 5 & Scheme 6 \\
\hline $\begin{array}{l}\text { Standard } \\
\text { BT design }\end{array}$ & $\begin{array}{l}\text { ND embedded standard } \\
\text { BT design, } r=10 \mathrm{~nm}\end{array}$ & $\begin{array}{l}\text { ND embedded standard } \\
\text { BT design, } r=15 \mathrm{~nm}\end{array}$ & $\begin{array}{l}2 \text { NDs embedded standard } \\
\text { BT design, } r=15 \mathrm{~nm}\end{array}$ & $\begin{array}{l}\text { NW embedded } \\
\text { standard BT design }\end{array}$ & $\begin{array}{l}\text { NW embedded } \\
\text { modified BT design }\end{array}$ \\
\hline $\begin{array}{l}w=50 \mathrm{~nm}, \\
L=350 \mathrm{~nm}, \\
d=30 \mathrm{~nm}, \\
g=30 \mathrm{~nm}\end{array}$ & $\begin{array}{l}w=50 \mathrm{~nm}, \\
L=350 \mathrm{~nm}, \\
d=30 \mathrm{~nm}, \\
g=40 \mathrm{~nm}\end{array}$ & $\begin{array}{l}w=50 \mathrm{~nm}, \\
L=350 \mathrm{~nm}, \\
d=30 \mathrm{~nm}, \\
g=40 \mathrm{~nm}\end{array}$ & $\begin{array}{l}w=50 \mathrm{~nm}, \\
L=350 \mathrm{~nm}, \\
d=30 \mathrm{~nm}, \\
g 1=g 3=3.5 \mathrm{~nm}, g 2=3 \mathrm{~nm}\end{array}$ & $\begin{array}{l}d=30 \mathrm{~nm}, \\
L=350 \mathrm{~nm}, \\
g 1=5 \mathrm{~nm}, \\
g 2=5 \mathrm{~nm}, H_{\mathrm{NW}}=30 \mathrm{~nm}\end{array}$ & $\begin{array}{l}d=30 \mathrm{~nm}, \\
L=350 \mathrm{~nm}, \\
g 1=5 \mathrm{~nm}, \\
g 2=5 \mathrm{~nm}, H_{\mathrm{NW}}=50 \mathrm{~nm}\end{array}$ \\
\hline
\end{tabular}

The real part of $n_{\text {eff }}$ is directly dependent on the SPP mode confinement in the cavity as shown in Fig. 4(a). The $n_{\text {eff }}$ of the SPP resonant mode is defined as $\beta / \beta_{o}$ which is dependent on the $\mathrm{BT}$ configuration, where $\beta$ is the propagation constant in the MIM waveguide and $\beta_{o}$ is the free space propagation constant. As the mode is squeezed in the cavity, the $n_{\text {eff }}$ increases. For instance, the standard BT design has $n_{\text {eff }}=1.1517$ which increases to
2.0129 when NW of $H_{\mathrm{NW}}=50 \mathrm{~nm}$ and $W_{\mathrm{NW}}=20 \mathrm{~nm}$ is introduced as shown in Fig. 4(a). Among all, Scheme 6 has the highest $n_{\text {eff }}$ which is highly responsive to the slight change in the ambient refractive index as displayed in Fig. 4(b). The NW embedded modified BT design is highly attractive for refractive index sensing applications as its $S_{\mathrm{SPP}}$ is 3.5 which is $50 \%$ higher than that of the standard BT design. 


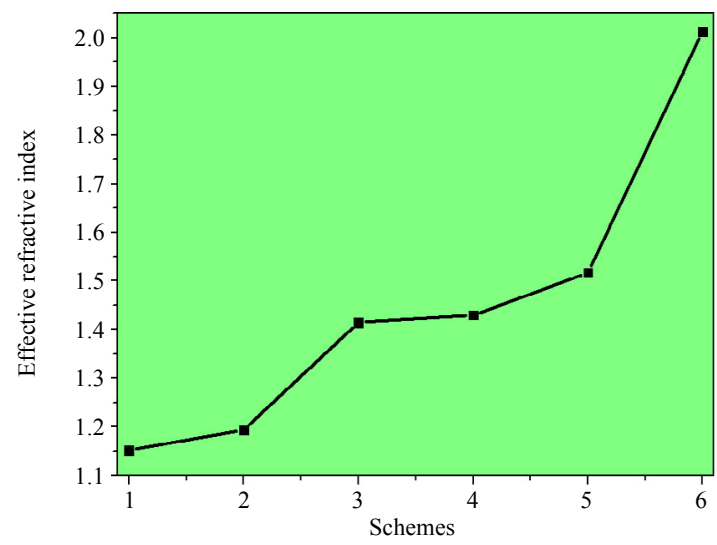

(a)

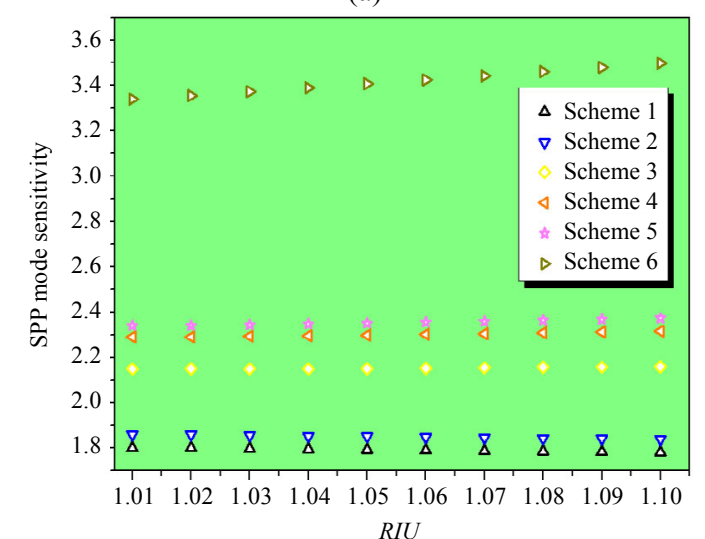

(b)

Fig. 4 Effective refractive index and mode sensitivity analysis (a) real part of $n_{\text {eff }}$ of the SPP mode and ( b) SPP mode sensitivity.

\section{Results and discussion}

SPs are exceedingly sensitive to the changes in the ambient refractive index. The measurement of $\Delta \lambda_{\text {res }}$ is the most widespread interrogation method used in ring resonators based on plasmonic structures [35]. This is often quantified as the bulk refractive index sensitivity $(S)$, defined as $S=$ $\Delta \lambda_{\text {res }} / \Delta n$, where $\lambda_{\text {res }}$ is the wavelength at which the excitation of SP occurs and $\Delta n$ is the change in the RI. For sensing application, FOM is usually applied to further assess the sensing performance and is expressed as $F O M=S / F W H M$ where FWHM is the full width at half maximum. As a sensor, both high $S$ and FOM are essential to assuring an outstanding performance with high optical resolution. A lot of research has been done to enhance the $S$ of plasmonic sensors. One of the major problems in plasmonics is the large line width of plasmon resonances due to the large radiative damping of the metal which results in lower FOM and $Q$-factor. In reality, FWHM is connected to the SP lifetime (or propagation length) which typically controls the sharpness of the peak. The FOM is perhaps the most general metric for comparing the performance of plasmonic platforms. The $Q$-factor is defined as $\lambda_{\text {res }}$ $F W H M$, where $\lambda_{\text {res }}$ is the resonance wavelength. The spectral response of the sensor is calculated by filling the medium with several refractive indices. The normalized intensity at the output bus waveguide is calculated in the NIR wavelength range for $n=1.0,1.02,1.04,1.06,1.08$, and 1.1. The $S, F O M$, and $Q$-factor are calculated for six different design schemes as shown in Figs. 5(a) and 5(b).

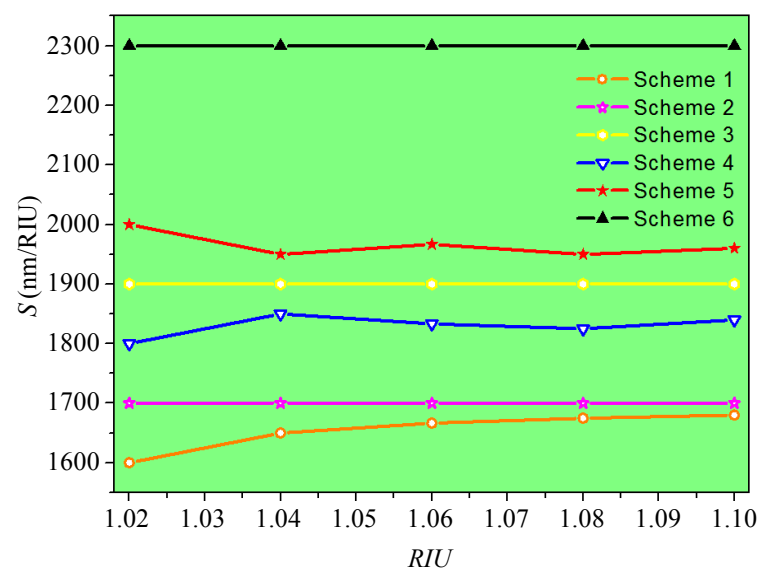

(a)

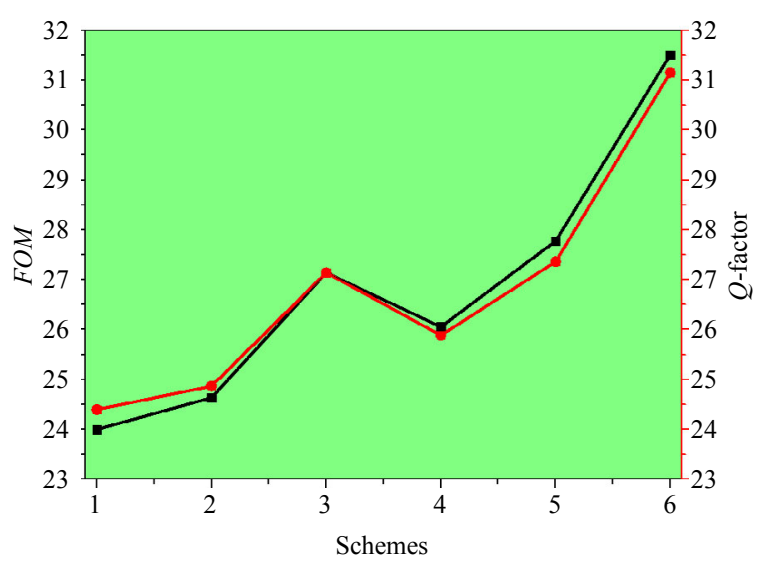

(b)

Fig. 5 Sensor performance: (a) $S$ and (b) FOM and $Q$-factor of design Schemes $1-6$. 
The spectral characteristics of six BT design schemes are presented in Table 3. Among all the proposed BT configurations, Sheme 6 offers the highest $S, F O M$ and $Q$-factor of $2300 \mathrm{~nm} / \mathrm{RIU}$, 31.5, and 31.1, respectively, as presented in Figs.5(a) and 5(b). Whereas, all six proposed BT sensor schemes offer comparable or even higher $S$ than several previously reported sensor designs [36-47] as displayed in Fig. 6.

Table 3 Spectral characteristics of proposed sensor designs based on the BT configuration.

\begin{tabular}{cccccccc}
\hline $\begin{array}{c}\text { BT resonator } \\
\text { configuration }\end{array}$ & Scheme & Scheme & Scheme & Scheme & Scheme & Scheme \\
\hline$\lambda_{\text {res }}(\mathrm{nm})$ & 1708 & 1716 & 1900 & 1838 & 1970 & 2274 \\
$S(\mathrm{~nm} / \mathrm{RIU})$ & 1680 & 1700 & 1900 & 1850 & 2000 & 2300 \\
$F O M$ & 24 & 24.6 & 27.1 & 26 & 27.8 & 31.5 \\
$Q$-factor & 24.4 & 24.9 & 27.2 & 25.9 & 27.4 & 31.1 \\
\hline
\end{tabular}

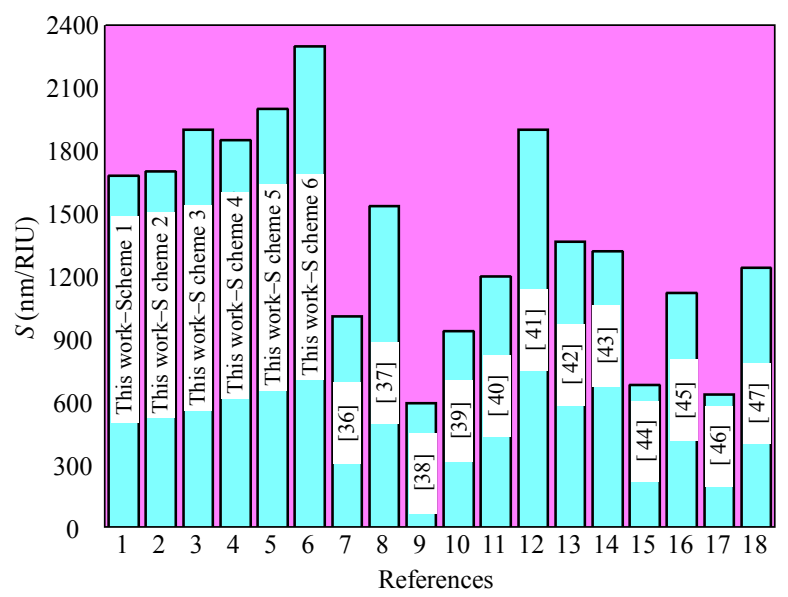

Fig. 6 Sensitivity comparison between the proposed BT configurations and previously proposed works.

The fabrication of the proposed sensor design is a flexible process and can be achieved in a few simple steps. To start with, a thin layer of silver is deposited on a silica substrate followed by E-beam lithography patterning of the design. The main advantage of E-beam lithography is due to its ability to inscribe custom patterns with sub-10 nm resolution [48]. To obtain MIM waveguides, it is important to remove the undesirable parts of silver metal from the design with the help of chemical etching. One potential method is the use of diluted nitric acid and water which offers the high etch selectivity.

\section{Conclusions}

In this work, we investigate six different configurations of the Bow Tie resonator designs using the finite element method. The influence of geometric parameters of the sensor on the resonance wavelength is also comprehensively studied. The sensitivity of the resonant mode can be increased by narrowing down the cavity region where the surface plasmon polariton is confined. The mode sensitivity analysis suggests that the nanowall embedded cavity design is highly sensitive to the refractive index change in the ambient medium. The device sensitivity of the standard Bow Tie cavity design is attained at $1680 \mathrm{~nm} / \mathrm{RIU}$ which is further improved by incorporating nanowall in the center of the modified Bow Tie cavity design. The maximum sensitivity, the figure of merit, and the quality factor of the nanowall embedded modified Bow Tie cavity is $2300 \mathrm{~nm} / \mathrm{RIU}, 31.5$, and 31.1, respectively. We believe that this study will provide new opportunities in the realization of nanowall embedded plasmonic sensors for the measurement of solution concentration and $\mathrm{pH}$ values of the biological and chemical samples.

\section{Acknowledgment}

This work was financially supported by the Ministry of Science and Higher Education within the State assignment FSRC "Crystallography and Photonics" RAS (Grant No. 007-GZ/Ch3363/26).

Open Access This article is distributed under the terms of the Creative Commons Attribution 4.0 International License (http://creativecommons.org/ licenses/by/4.0/), which permits unrestricted use, distribution, and reproduction in any medium, provided you give appropriate credit to the original author(s) and the source, provide a link to the Creative Commons license, and indicate if changes were made. 


\section{References}

[1] W. L. Barnes, A. Dereux, and T. W. Ebbesen, "Surface plasmon subwavelength optics," Nature, 2003, 424(6950): 824-830.

[2] B. Hecht, H. Bielefeldt, L. Novotny, Y. Inouye, and D. W. Pohl, "Local excitation, scattering, and interference of surface plasmons," Physical Review Letters, 1996, 77(9): 1889.

[3] C. I. Lin and T. K. Gaylord, "Multimode metal-insulator-metal waveguides: analysis and experimental characterization," Physical Review B, 2012, 85(8): 085405.

[4] M. Singh and S. K. Rughuwanshi, "Metal-insulator-metal waveguide based passive structures analyzed by transmission line model," Superlattices and Microstructures, 2018, 114: 233-241.

[5] J. Lin, J. Dellinger, P. Genevet, B. Cluzel, F. D. Fornel, and F. Capasso, "Cosine-Guass plasmon beam: a localized long range-nondiffracting surface wave," Physical Review Letters, 2012, 109(9): 093904.

[6] M. A. Izadi and R. Nouroozi, "Adjustable propagation length enhancement of the surface plasmon polariton wave via phase sensitive optical parametric amplification," Scientific Reports, 2018, 8(1): 1-14.

[7] C. H. Du and Y. P. Chiou, "Vertical directional couplers with ultra-short coupling length based on hybrid plasmonic waveguides," Journal of Lightwave Technology, 2014, 32(11): 2065-2071.

[8] B. Yun, G. Hu, and Y. Cui, "Theoretical analysis of a nanoscale plasmonic filter based on a rectangular metal-insulator-metal waveguide," Journal of Physics D: Applied Physics, 2010, 43(38): 385102.

[9] S. Khani, M. Danaie, and P. Rezaei, "Size reduction of MIM surface plasmon based optical bandpass filters by the introduction of arrays of silver nano-rods," Physica E: Low-Dimensional Systems and Nanostructures, 2019, 113: 25-34.

[10] M. A. Butt, S. N. Khonina, and N. L. Kazanskiy, "Highly sensitive refractive index sensor based on hybrid plasmonic waveguide microring resonator," Waves in Random and Complex Media, 2020, 30(2): 292-299.

[11] M. A. Butt, S. N. Khonina, and N. L. Kazanskiy, "Hybrid plasmonic waveguide-assisted metalinsulator-metal ring resonator for refractive index sensing," Journal of Modern Optics, 2018, 65(9): 1135-1140.

[12] M. A. Butt, S.N. Khonina, and N. L. Kazanskiy, “A multichannel metallic dual nano-wall square split-ring resonator: design analysis and applications," Laser Physics Letters, 2019, 16: 126201

[13] Y. Y. Xie, C. He, J. C. Li, T. T. Song, Z. D. Zhang, and Q. R. Mao, "Theoretical investigation of a plasmonic demultiplexer in MIM waveguide crossing with multiple side-coupled hexagonal resonators," IEEE Photonics Journal, 2016, 8(5): 1-12.

[14] S. Qu, C. Ma, and H. Liu, "Tunable graphene-based hybrid plasmonic modulators for subwavelength confinement," Scientific Reports, 2017, 7(1): 1-8.

[15] A. N. Taheri and H. Kaatuzian, "Design and simulation of a nanoscale electro-plasmonic $1 \times 2$ switch based on asymmetric metal-insulator-metal stub filters," Applied Optics, 2014, 53(28): 6546-6553.

[16] C. H. Chen and K. S. Liao, " $1 \times N$ plasmonic power splitters based on metal-insulator-metal waveguides," Optics Express, 2013, 21(4): 4036-4043.

[17] P. Vaiano, B. Carotenuto, M. Pisco, A. Ricciardi, G. Quero, M. Consales, et al., "Lab on fiber technology for biological sensing applications," Laser \& Photonics Reviews, 2016, 10(6): 922-961.

[18] X. Cheng, J. Bonefacino, B. O. Guan, and H. Y. Tam, "All-polymer fiber-optic pH sensor," Optics Express, 2018, 26(11): 14610-14616.

[19] S. Wang, K. Broderick, H. Smith, and Y. Yi, "Strong coupling between on chip notched ring resonator and nanoparticle," Applied Physics Letters, 2010, 97(5): 051102.

[20] Z. D. Zhang, H. Y. Wang, and Z. Y. Zhang, "Fano resonance in a gear-shaped nanocavity of the metal-insulator-metal waveguide," Plasmonics, 2013, 8(2): 797-801.

[21] Z. Guo, K. Wen, Q. Hu, W. Lai, J. Lin, and Y. Fang, "Plasmonic multichannel refractive index sensor based on subwavelength tangent-ring metal-insulator-metal waveguide," Sensors, 2018, 18(5): 1348

[22]Z. Zhang, J. Yang, X. He, J. Zhang, J. Huang, D. Chen, et al., "Plasmonic refractive index sensor with high figure of merit based on concentric-rings resonator," Sensors, 2018, 18(1): 116.

[23] N. L. Kazanskiy and M. A. Butt, "Enhancing the sensitivity of a standard plasmonic MIM square ring resonator by incorporating nano-dots in the cavity," Photonics Letters of Poland, 2020, 12(1): 1-3.

[24] Y. Y. Xie, Y. X. Huang, W. L. Zhao, W. H. Xu, and C. $\mathrm{He}$, "A novel plasmonic sensor based on metal-insulator-metal waveguide with a side-coupled hexagonal cavity," IEEE Photonics Journal, 2015, 7(2): 1-12.

[25] S. Wang, S. Yu, T. Zhao, Y. Wang, and X. Shi, "A nanosensor with ultra-high FOM based on tunable malleable multiple Fano resonances in a waveguide coupled isosceles triangular resonator," Optics Communications, 2020, 465: 125614.

[26] S. Yan, M. Zhang, X. Zhao, Y. Zhang, J. Wang, and W. Jin, "Refractive index sensor based on a metal-insulator-metal waveguide coupled with a symmetric structure," Sensors, 2017, 17(12): 2879.

[27] X. Zhao, Z. Zhang, and S. Yan, "Tunable Fano 
resonance in asymmetric MIM waveguide structure," Sensors, 2017, 17(7): 1494.

[28] Y. Zhang, Y. Kuang, Z. Zhang, Y. Tang, J. Han, R. Wang, et al., "High-sensitivity refractive index sensors based on Fano resonance in the plasmonic system of splitting ring cavity-coupled MIM waveguide with tooth cavity," Applied Physics A, 2019, 125(13): 1-5.

[29] Y. Zhang and M. Cui, "Refractive index sensor based on the symmetric MIM waveguide structure," Journal of Electronic Materials, 2019, 48(2): 1005-1010.

[30] S. B. Yan, L. Luo, C. Y. Xue, and Z. D. Zhang, "A refractive index sensor based on a metalinsulator-metal waveguide-coupled ring resonator," Sensors, 2015, 15(11): 29183-29191.

[31] P. C. Wu, G. Sun, W. T. Chen, K. Y. Yang, Y. W. Huang, Y. H. Chen, et al., "Vertical split-ring resonator based nanoplasmonic sensor," Applied Physics Letters, 2014, 105(3): 033105.

[32] M. A. Butt, S. N. Khonina, and N. L. Kazanskiy, "A multichannel metallic dual nano-wall square split-ring resonator: design analysis and applications," Laser Physics Letters, 2019, 16(12): 126201.

[33] S. Yu, T. Zhao, J. Yu, and D. Pan, "Tuning multiple fano resonances for on-chip sensors in a plasmonic system," Sensors, 2019, 19(7): 1559.

[34] P. D. Sia, "Overview of Drude-Lorentz type models and their applications," Nanoscale Systems: Mathematical Modeling, Theory and Applications, 2014, 3(1): 1-13.

[35] J. Homola, S. Sinclair, and G. Gauglitz, "Surface plasmon resonance sensors: review," Sensors and Actuators B: Chemical, 1999, 54(1-2): 3-15.

[36] W. Wei, X. Zhang, and X. Ren, "Plasmonic circular resonators for refractive index sensors and filters," Nanoscale Research Letters, 2015, 10(1): 211.

[37] S. Gaur, R. Zafar, and D. Somwanshi, "Plasmonic refractive index sensor based on metal insulator metal waveguide," IEEE International Conference on Recent Advances and Innovations in Engineering (ICRAIE-2014), India, Dec. 23-35, pp. 1-4, 2016.

[38] Z. Zhang, L. Luo, C. Xue, W. Zhang, and S. Yan, "Fano resonance based on metal-insulator-metal waveguide-coupled double rectangular cavities for plasmonic nanosensors," Sensors, 2016, 16(5): 642.

[39] B. F. Yun, G. H. Hu, R. H. Zhang, and Y. P. Cui, "Fano resonances in a plasmonic waveguide system composed of stub coupled with a square cavity resonator," Journal of Optics, 2016, 18(5): 055002.

[40] M. A. Butt, S. N. Khonina, and N. L. Kazanskiy, "Plasmonic refractive index sensor based on MIM square ring resonator," 2018 International Conference on Computing, Electronic and Electrical Engineering (ICE Cube), Pakistan, Nov. 12-13, pp. $1-4,2018$.

[41] S. Asgari and N. Granpayeh, "Tunable mid-infrared refractive index sensor composed of asymmetric double graphene layers," IEEE Sensors Journal, 2019, 19(14): 5686-5691.

[42]M. A. Butt, S. N. Khonina, and N. L. Kazanskiy, "Plasmonic refractive index sensor based on metal-insulator-metal waveguides with high sensitivity," Journal of Modern Optics, 2019, 66(9): 1038-1043.

[43] M. A. Butt, S. N. Khonina, and N. L. Kazanskiy, "Metal-insulator-metal nano square ring resonator for gas sensing applications," Waves in Random and Complex Media, 2019, DOI.org/10.1080/17455030.2019.1568609.

[44] L. Wang, Y. P. Zeng, Z. Y. Wang, X. P. Xia, and Q. Q. Liang, "A refractive index sensor based on an analogy T shaped metal-insulator-metal waveguide," Optik, 2018, 172: 1199-1204.

[45] Y. Chen, Y. Xu, and J. Cao, "Fano resonance sensing characteristics of MIM waveguide coupled square convex ring resonator with metallic baffle," Results in Physics, 2019, 14: 102420.

[46] M. Danaie and A. Shahzadi, "Design of a high-resolution metal-insulator-metal plasmonic refractive index sensor based on a ring-shaped $\mathrm{Si}$ resonator," Plasmonics, 2019, 14: 1453-1465.

[47] M. A. Butt, S. N. Khonina, and N. L. Kazanskiy, “An array of nano-dots loaded MIM square ring resonator with enhanced sensitivity at NIR wavelength range," Optik, 2020, 202: 163655.

[48] M. Feldman, Nanolithography: the art of fabricating nanoelectronic and nanophotonic devices and systems. England: Woodhead Publishing, 2014. 